\title{
On a Dirichlet to Neumann and Robin to Neumann operators suitable for reflecting harmonic functions subject to a nonhomogeneous condition on an arc
}

\author{
Murdhy Aldawsari Tatiana Savina
}

January 7, 2019

\begin{abstract}
According to the Schwarz symmetry principle, every harmonic function vanishing on a real analytic curve has an odd continuation, while a harmonic function satisfying homogeneous Neumann condition has the even continuation. There are different generalizations of the Schwarz symmetry principle. Most of them are dealing with homogeneous conditions and the Dirichlet case. Using a technique of Dirichlet to Neumann and Robin to Neumann operators, we derive reflection formulae for nonhomogeneous Neumann and Robin conditions from a reflection formula subject to a nonhomogeneous Dirichlet condition.

Keywords: Schwarz symmetry principle, Dirichlet to Neumann operator, Robin to Neumann operator, Analytic continuation
\end{abstract}

\section{Introduction}

Let $\Gamma \subset \mathbb{R}^{2}$ be a non-singular real analytic curve and a point $z_{0}=\left(x_{0}, y_{0}\right) \in \Gamma$. Then, there exists a neighborhood $\Omega$ of $z_{0}$ and an anti-conformal mapping $R: \Omega \rightarrow \Omega$ which is identity on $\Gamma$, permutes the components $\Omega_{1}, \Omega_{2}$ of $\Omega \backslash \Gamma$ and relative to which any harmonic function $u(x, y)$ defined near $\Gamma$ and vanishing on $\Gamma$ (the homogeneous Dirichlet condition) is odd,

$$
u(x, y)=-u(R(x, y)) .
$$

In the case of nonhomogeneous Dirichlet data, $u=\varphi(x, y)$ on $\Gamma$, when function $\varphi$ is holomorphically continuable into $\mathbb{C}^{2}$ near $\Gamma$, formula (1) involves also values of function $u$ at two additional points located on the complexification $\Gamma_{\mathbb{C}}$ of the curve $\Gamma$. All four points then create a so-called Study's rectangle [6].

To describe the Study's rectangle, consider a complex domain $W$ in the space $\mathbb{C}^{2}$ to which the function $f$ defining the curve $\Gamma:=\{f(x, y)=0\}$ can be analytically continued such that $W \cap \mathbb{R}^{2}=U$. Using the change of variables $z=x+i y, \zeta=x-i y$, the 
equation of the complexified curve $\Gamma_{\mathbb{C}}$ can be rewritten in the form

$$
f\left(\frac{z+\zeta}{2}, \frac{z-\zeta}{2 i}\right)=0,
$$

and if $\operatorname{grad} f(x, y) \neq 0$ on $\Gamma$, can be also rewritten in terms of the Schwarz function and its inverse, $w=S(z)$ and $z=\widetilde{S}(\zeta)$ [2]. The mapping $R$ mentioned above can be expressed in terms of the Schwarz function as follows,

$$
R(x, y)=R(z)=\overline{S(z)} .
$$

Using the above notations, the reflection formula for harmonic functions subject to conditions $u_{\mid \Gamma}=\phi$ can be written as the Study's rectangle:

$$
u(z, \zeta)+u(\widetilde{S}(\zeta), S(z))=\varphi(\widetilde{S}(\zeta), \zeta)+\varphi(z, S(z))
$$

The motivation of this paper goes back to D. Khavinson's suggestion to think of a different method for deriving reflection formulae rather than using the reflected fundamental solution method, described, for example, in [3]. This suggestion perhaps was rather related to methods used in analysis. However, L. Beznea's talk, devoted to Dirichlet to Neumann operators, given at the International Conference on Complex Analysis, Potential Theory and Applications in honour of Professor Stephen J Gardiner on the occasion of his 60th Birthday, influenced this attempt to derive reflection formulas for other than Dirichlet conditions using similar to Dirichlet to Neumann operators studied by L. Beznea et al. [1].

The structure of the paper is as follows. In section 2 we discuss some preliminaries. In section 3 we discuss a Dirichlet to Neumann operator for a circular arc written in a form suitable for reflection. In section 4 we use this formula to derive a reflection formula for the case of nonhomogeneous Neumann conditions given on a circular arc. In section 5 we discuss a Robin to Neumann operator for a circular arc. Section 6 is devoted to reflection for the case of a nonhomogeneous Robin data on a circular arc, and section 7 discusses a Dirichlet to Neumann operator and reflection for a harmonic function subject to nonhomogeneous Neumann condition on an arc of an algebraic curve.

\section{Preliminaries}

Consider the Neumann boundary value problem in the unit disk $\mathbb{D}$ in the plane:

$$
\begin{cases}\Delta V=0 & \text { in } \quad \mathbb{D} \\ \frac{\partial V}{\partial n}=\varphi & \text { on } \quad \partial \mathbb{D}\end{cases}
$$

where $n$ is the outward normal. Solution to this problem can be written in terms of the solution to a corresponding Dirichlet problem, $V=\Lambda(U)[1]$. Here $U(x, y)$ is defined by

$$
\left\{\begin{array}{l}
\Delta U=0 \quad \text { in } \quad \mathbb{D} \\
U=\varphi \quad \text { on } \quad \partial \mathbb{D},
\end{array}\right.
$$

where $\varphi \in C(\partial \mathbb{D})$. 
Theorem 1. [1] Assume $\varphi: \partial \mathbb{D} \rightarrow \mathbb{R}$ is continuous and its integral along $\partial \mathbb{D}$ vanishes. If $u$ is the solution to the Dirichlet problem (5), then

$$
V(z)=\Lambda(U)=\int_{0}^{1} \frac{U(\rho z)}{\rho} d(\rho), \quad z \in \mathbb{D} \cup \partial \mathbb{D},
$$

is the solution to the Neumann problem (4) with $V(0)=0$, where $z=x+i y$.

A derivation of formula (6) in paper [1] was based on the following consideration. If $U(x, y)=\mathfrak{R}[g(z)]$ and $V(x, y)=\Re[f(z)]$, where $g(z)$ and $f(z)$ are analytic functions, then the following equalities hold on $\partial \mathbb{D}$,

$$
\varphi=U=\frac{\partial V}{\partial n}=(\nabla V \cdot n)=\frac{\partial V}{\partial x} x+\frac{\partial V}{\partial y} y=\Re\left[z f^{\prime}(z)\right]=\Re[g(z)] .
$$

Setting $z f^{\prime}(z)=g(z)$ and integrating with from $z$ to $z_{0}$, one has

$$
f(z)=f\left(z_{0}\right)-\int_{z}^{z_{0}} \frac{g(\tau)}{\tau} d \tau, \quad z, z_{0} \in \mathbb{D} \cup \partial \mathbb{D} .
$$

Formula (7) is a starting point for writing an expression relating harmonic functions satisfying Dirichlet, Neumann, and Robin conditions on $\partial \mathbb{D}$.

\section{Dirichlet to Neumann operator for an arc of the unit circle}

Consider the following two problems for functions $u(x, y)$ and $v(x, y)$ defined near an $\operatorname{arc} \partial \mathbb{D}$ of a unit circle in the plane:

$$
\begin{aligned}
& \begin{cases}\Delta u=0 & \text { near } \quad \partial \mathbb{D} \\
u=\varphi & \text { on } \partial \mathbb{D},\end{cases} \\
& \begin{cases}\Delta v=0 & \text { near } \quad \partial \mathbb{D} \\
\frac{\partial v}{\partial n}=\varphi & \text { on } \quad \partial \mathbb{D},\end{cases}
\end{aligned}
$$

where $\varphi$ is holomorphically continuable into $\mathbb{C}^{2}$ near $\partial \mathbb{D}$. To find and expression relating $v(x, y)$ and $u(x, y)$ one can take the real part of (7) by computing $v(x, y)=$ $\frac{1}{2}(f(z)+\overline{f(z)})$. However, for what follows it is more convenient to complexify the problem, considering functions $v(z, \zeta)$ and $u(z, \zeta)$, where $(z, \zeta) \in \mathbb{C}^{2}$ with $\zeta=\bar{z}$ on $\mathbb{R}^{2}$.

Theorem 2. Let $\partial \mathbb{D}$ be the unit circle in the plane centered at the origin. If $u(z, \zeta)$ is a solution to (8), then

$$
v(z, \zeta)=v\left(z_{0}, \zeta_{0}\right)-\int_{z}^{z_{0}} \frac{u_{1}(\tau)}{\tau} d \tau-\int_{\zeta}^{\zeta_{0}} \frac{u_{2}(\xi)}{\xi} d \xi
$$


is a solution to (9). Here $u(z, \zeta)=u_{1}(z)+u_{2}(\zeta)$ is a harmonic function such that $u(z, \bar{z})=\varphi$ on $\partial \mathbb{D}$.

Proof. To prove formula (10) one just needs to check the conditions in (9). Obviously, function $v(z, \zeta)$ is harmonic, $\frac{\partial^{2} v}{\partial z \partial \zeta}=0$.

To check the second condition in $(9)$, we fix a point $\left(x_{0}, y_{0}\right) \in \partial \mathbb{D}$. Using the notation $z_{0}=x_{0}+i y_{0}$, the outward normal at $z_{0}, n\left(z_{0}\right)=z_{0}$, can be computed as

$$
\frac{\partial v}{\partial n}\left(z_{0}\right)=\lim _{h \rightarrow 0} \frac{v\left(z_{0}+h z_{0}\right)-v\left(z_{0}\right)}{h}=\lim _{h \rightarrow 0} \frac{v\left(z_{0}(h+1)\right)-v\left(z_{0}\right)}{h} .
$$

Taking into account the expression (10), we have

$$
\frac{\partial v}{\partial n}\left(z_{0}\right)=\lim _{h \rightarrow 0} \frac{1}{h}\left(\int_{e^{i \theta}}^{(h+1) e^{i \theta}} \frac{u_{1}(\tau)}{\tau} d \tau+\int_{e^{-i \theta}}^{(h+1) e^{-i \theta}} \frac{u_{2}(\xi)}{\xi} d \xi\right) .
$$

Using the substitutions $\tau=r e^{i \theta}, \xi=r e^{-i \theta}$ with fixed $\theta$, the above formula yields

$$
\frac{\partial v}{\partial n}\left(z_{0}\right)=\lim _{h \rightarrow 0} \frac{1}{h} \int_{1}^{h+1} \frac{u_{1}\left(r e^{i \theta}\right)+u_{2}\left(r e^{-i \theta}\right)}{r} d r=\lim _{\widetilde{r} \rightarrow 1} \frac{u\left(\widetilde{r} e^{i \theta}, \widetilde{r} e^{-i \theta}\right)}{\widetilde{r}}=u\left(e^{i \theta}, e^{-i \theta}\right)=\varphi\left(x_{0}, y_{0}\right) .
$$

This finishes the proof.

Example 3.1. Let harmonic function satisfying the Dirichlet condition $\varphi=C$ be $u(x, y)=$ $C$, where $C$ is a constant. Then for function $v(x, y)$, we have,

$$
v(z, \zeta)=v\left(z_{0}, \zeta_{0}\right)-\frac{1}{2} \int_{z}^{z_{0}} \frac{C}{\tau} d \tau-\frac{1}{2} \int_{\zeta}^{\zeta_{0}} \frac{C}{\xi} d \xi,
$$

which implies that the corresponding to $u(x, y)=C$ function, satisfying Neumann condition $\frac{\partial v}{\partial n}=C$, is $v(x, y)=C \ln \sqrt{x^{2}+y^{2}}+$ const.

Example 3.2. Let harmonic function satisfying homogeneous Dirichlet condition be $u(x, y)=\ln \sqrt{x^{2}+y^{2}}=\frac{1}{2} \ln z+\frac{1}{2} \ln \zeta$. Let us compute the corresponding function $v(x, y)$ with $\varphi=0$,

$$
v(z, \zeta)=v\left(z_{0}, \zeta_{0}\right)-\frac{1}{2} \int_{z}^{z_{0}} \frac{\ln \tau}{\tau} d \tau-\frac{1}{2} \int_{\zeta}^{\zeta_{0}} \frac{\ln \xi}{\xi} d \xi,
$$

which implies that the corresponding solution of problem (9) is

$$
v(x, y)=\frac{1}{2}\left(\ln ^{2} \sqrt{x^{2}+y^{2}}-\arctan ^{2} \frac{y}{x}\right)+\text { const } .
$$

The latter expression in polar coordinates, $z=r e^{i \theta}$, has a simpler form, $v(r, \theta)=$ $\frac{1}{4}\left(\ln ^{2} r-\theta^{2}\right)+$ const. One can easily check that $v(r, \theta)$ satisfies the Laplace's equation, $\Delta v=\frac{\partial^{2} v}{\partial r^{2}}+\frac{1}{r} \frac{\partial v}{\partial r}+\frac{1}{r^{2}} \frac{\partial^{2} v}{\partial \theta^{2}}$, and its normal derivative vanishes on the unit circle. 
Example 3.3. Harmonic function $u(x, y)=x^{2}-y^{2}$ on a unit circle equals to $\varphi=$ $2 x^{2}-1=\cos (2 \theta)$. To find the corresponding functions $u_{1}(z)$ and $u_{2}(\zeta)$, we make the substitution $x=(z+\zeta) / 2$ and $y=(z-\zeta) /(2 i)$. This results in $u_{1}=z^{2} / 2$ and $u_{2}=\zeta^{2} / 2$. Thus the corresponding Dirichlet to Neumann operator is

$$
v(z, \zeta)=v\left(z_{0}, \zeta_{0}\right)-\frac{1}{2} \int_{z}^{z_{0}} \frac{\tau^{2}}{\tau} d \tau-\frac{1}{2} \int_{\zeta}^{\zeta_{0}} \frac{\xi^{2}}{\xi} d \xi .
$$

Thus, $v(x, y)=\frac{1}{2}\left(x^{2}-y^{2}\right)+$ const, which is obviously a harmonic function. This function in polar coordinates has the form $v(r, \theta)=\frac{1}{2} r^{2} \cos (2 \theta)$, whose normal derivative on the unit circle is $(\nabla v \cdot n)=\cos (2 \theta)$, which equals to $\varphi$.

Example 3.4. Harmonic function $u(x, y)=x$ on a unit circle equals to $\varphi=\cos \theta$. The corresponding functions $u_{1}=z / 2$ and $u_{2}=\zeta / 2$. Thus the expression for $v$ is

$$
v(z, \zeta)=v\left(z_{0}, \zeta_{0}\right)-\frac{1}{2} \int_{z}^{z_{0}} \frac{\tau}{\tau} d \tau-\frac{1}{2} \int_{\zeta}^{\zeta_{0}} \frac{\xi}{\xi} d \xi
$$

therefore, $v(x, y)=x+$ const, which is obviously a harmonic function. This function in polar coordinates has the form $v(r, \theta)=\frac{1}{2} r^{2} \cos (2 \theta)$, whose normal derivative on the unit circle equals to $\varphi,(\nabla v \cdot n)=\cos \theta$.

\section{Reflection about an arc of a circle with nonhomoge- neous Neumann data}

Consider Neumann problem (9) in a neighborhood $\Omega$ of a point $\left(z_{0}, \bar{z}_{0}\right) \in \partial \mathbb{D}$. Let point $z \in \Omega_{1} \subset \Omega$ for which formula (10) holds,

$$
v(z, \zeta)=v\left(z_{0}, \zeta_{0}\right)-\int_{z}^{z_{0}} \frac{u_{1}(\tau)}{\tau} d \tau-\int_{\zeta}^{\zeta_{0}} \frac{u_{2}(\xi)}{\xi} d \xi .
$$

For what follows, it is convenient to fix $\theta$ and to rewrite the integrals in polar coordinates,

$$
v(z, \zeta)=v\left(z_{0}, \zeta_{0}\right)-\int_{r}^{1} \frac{u\left(\rho e^{i \theta}, \rho e^{-i \theta}\right)}{\rho} d \rho
$$

where $r=|z|$.

We remark that formula (3) for the unite circle can be rewritten as

$$
u\left(r e^{i \theta}, \frac{r}{e^{i \theta}}\right)+u\left(\frac{e^{i \theta}}{r}, \frac{1}{r e^{i \theta}}\right)=\varphi\left(r e^{i \theta}, \frac{1}{r e^{i \theta}}\right)+\varphi\left(\frac{e^{i \theta}}{r}, \frac{r}{e^{i \theta}}\right) .
$$


Applying reflection, $R: \Omega \rightarrow \Omega$, to formula (11) and taking into account that the boundary points are fixed under $R$, we have

$$
v(R(z, \zeta))=v\left(z_{0}, \zeta_{0}\right)+I_{1}+I_{2}+I_{3},
$$

where

$$
I_{1}=\int_{\frac{1}{r}}^{1} \frac{u\left(\frac{e^{i \theta}}{\rho}, \frac{1}{\rho e^{i \theta}}\right)}{\rho} d \rho, \quad I_{2}=-\int_{\frac{1}{r}}^{1} \frac{\varphi\left(\rho e^{i \theta}, \frac{1}{\rho e^{i \theta}}\right)}{\rho} d \rho, \quad I_{3}=-\int_{\frac{1}{r}}^{1} \frac{\varphi\left(\frac{e^{i \theta}}{\rho}, \frac{\rho}{e^{i \theta}}\right)}{\rho} d \rho .
$$

To obtain a reflection formula for Neumann conditions, we need to rewrite the right hand side of (13) in terms of $v(z, \zeta)$. In order to do that, we make a substitution $\tilde{\rho}=1 / \rho$ in $I_{1}$, which results in

$$
I_{1}=\int_{\frac{1}{r}}^{1} \frac{u\left(\frac{e^{i \theta}}{\rho}, \frac{1}{\rho e^{i \theta}}\right)}{\rho} d \rho=-\int_{r}^{1} \frac{u\left(\tilde{\rho} e^{i \theta}, \tilde{\rho} e^{-i \theta}\right)}{\tilde{\rho}} d \tilde{\rho}
$$

therefore,

$$
v\left(z_{0}, \zeta_{0}\right)+I_{1}=v(z, \zeta)
$$

The second and third integrals could be combined if we do the same substitution, $\tilde{\rho}=$ $1 / \rho$ in $I_{3}$. Indeed,

$$
I_{3}=-\int_{1}^{r} \frac{\varphi\left(\tilde{\rho} e^{i \theta}, \frac{1}{\tilde{\rho} e^{i \theta}}\right)}{\tilde{\rho}} d \tilde{\rho}
$$

hence,

$$
I_{2}+I_{3}=-\int_{\frac{1}{r}}^{r} \frac{\varphi\left(\rho e^{i \theta}, \frac{1}{\rho e^{i \theta}}\right)}{\rho} d \rho .
$$

Thus, we finally derived the following reflection formula for for harmonic functions satisfying Neumann condition on an arc of the unite circle

$$
v\left(\frac{e^{i \theta}}{r}, \frac{1}{r e^{i \theta}}\right)=v\left(r e^{i \theta}, r e^{-i \theta}\right)-\int_{\frac{1}{r}}^{r} \frac{\varphi\left(\rho e^{i \theta}, \frac{1}{\rho e^{i \theta}}\right)}{\rho} d \rho .
$$

Example 4.1. Consider a harmonic function, whose normal derivative on the unite circle, $x^{2}+y^{2}=1$, is constant, $\varphi=C$. Then formula (14) reads as

$$
v\left(\frac{e^{i \theta}}{r}, \frac{1}{r e^{i \theta}}\right)=v\left(r e^{i \theta}, r e^{-i \theta}\right)-2 C \ln r .
$$

Example 4.2. Consider a harmonic function, whose normal derivative on the unite circle, $x^{2}+y^{2}=1$, equals $\varphi=4 x^{2}-2$. To compute the integral in formula (14), we rewrite function $\varphi$ as

$$
\varphi=4 x^{2}-2=4\left(\frac{z+\zeta}{2}\right)^{2}-2=z^{2}+\zeta^{2}+2(z \zeta-1) .
$$


The last term vanishes on the complexification of the unit circle, therefore, $\varphi(z, \zeta)=$ $z^{2}+\zeta^{2}$, which implies $\varphi\left(\rho e^{i \theta}, \frac{1}{\rho e^{i \theta}}\right)=\rho^{2} e^{2 i \theta}+\frac{1}{\rho^{2} e^{2 i \theta}}$. Thus,

$$
-\int_{\frac{1}{r}}^{r} \frac{\varphi\left(\rho e^{i \theta}, \frac{1}{\rho e^{i \theta}}\right)}{\rho} d \rho=\int_{r}^{\frac{1}{r}} \frac{\rho^{2} e^{2 i \theta}+\frac{1}{\rho^{2} e^{i \theta}}}{\rho} d \rho .
$$

Then formula (14) reads as

$$
v\left(\frac{e^{i \theta}}{r}, \frac{1}{r e^{i \theta}}\right)=v\left(r e^{i \theta}, r e^{-i \theta}\right)+\left(\frac{1}{r^{2}}-r^{2}\right) \cos 2 \theta .
$$

\section{Neumann to Robin operator for an arc of the unit circle}

Consider the following Robin problem

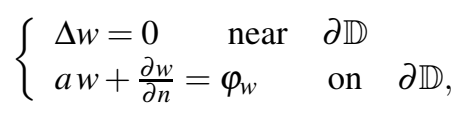

where $a$ and $b$ are real constants with $b \neq 0$.

Let $u$ and $v$ be harmonic functions defined near the arc $\partial \mathbb{D}$ of a unit circle in the plane:

$$
\begin{aligned}
& \begin{cases}\Delta u=0 & \text { near } \partial \mathbb{D} \\
u=\varphi_{u} & \text { on } \partial \mathbb{D},\end{cases} \\
& \left\{\begin{array}{llll}
\Delta v & =0 & \text { near } & \partial \mathbb{D} \\
\frac{\partial v}{\partial n}=\varphi_{v} & \text { on } & \partial \mathbb{D} .
\end{array}\right.
\end{aligned}
$$

Here $\varphi_{w}, \varphi_{u}$, and $\varphi_{v}$ are holomorphically continuable into $\mathbb{C}^{2}$ near $\partial \mathbb{D}$.

To derive a formula connecting functions $w(x, y), u(x, y)$, and $v(x, y)$, we think of them as real parts of analytic functions, $w=\mathfrak{R}[h], u=\mathfrak{R}[g]$, and $v=\mathfrak{R}[f]$ and assume that $\varphi_{w}=\varphi_{u}+\varphi_{v}$.

For a point on a boundary, $z_{0} \in \partial \mathbb{D}$, we have the following equalities

$$
\begin{gathered}
\frac{\partial v}{\partial n}\left(z_{0}\right)=D v \cdot n\left(z_{0}\right)=\mathfrak{R}\left[z_{0} f^{\prime}\left(z_{0}\right)\right]=\varphi_{v}, \\
a w+\frac{\partial w}{\partial n}\left(z_{0}\right)=a w+D w \cdot n\left(z_{0}\right)=\mathfrak{R}\left[a h+b z_{0} h^{\prime}\left(z_{0}\right)\right]=\varphi_{w} .
\end{gathered}
$$

Thus, setting

$$
a h(z)+b z h^{\prime}(z)=z f^{\prime}(z)+g(z)
$$

does not contradict the condition $\varphi_{w}=\varphi_{u}+\varphi_{v}$. Multiplying (20) by $z^{a / b-1} / b$, we have

$$
z^{\frac{a}{b}} h^{\prime}(z)+\frac{a}{b} z^{\frac{a}{b}-1} h(z)=\frac{1}{b} z^{\frac{a}{b}} f^{\prime}(z)+\frac{1}{b} z^{\frac{a}{b}-1} g(z),
$$


which can be rewritten as

$$
\left(z^{\frac{a}{b}} h(z)\right)^{\prime}=\frac{1}{b}\left(z^{\frac{a}{b}} f(z)\right)^{\prime}+\frac{1}{b} z^{\frac{a}{b}-1} g(z)-\frac{a}{b^{2}} z^{\frac{a}{b}-1} f(z) .
$$

Integrating from $z$ to $z_{0}$ along a segment with constant argument, we obtain

$$
z_{0}^{\frac{a}{b}} h\left(z_{0}\right)-z^{\frac{a}{b}} h(z)=\frac{1}{b}\left(z_{0}^{\frac{a}{b}} f\left(z_{0}\right)-z^{\frac{a}{b}} f(z)\right)+\frac{1}{b} \int_{z}^{z_{0}} \zeta^{\frac{a}{b}-1}\left[g(\zeta)-\frac{a}{b} f(\zeta)\right] d \zeta,
$$

which implies

$$
h(z)=\left(\frac{z_{0}}{z}\right)^{\frac{a}{b}}\left(h\left(z_{0}\right)-\frac{1}{b} f\left(z_{0}\right)\right)+\frac{1}{b} f(z)-\frac{1}{b} z^{-\frac{a}{b}} \int_{z}^{z_{0}} \zeta^{\frac{a}{b}-1}\left[g(\zeta)-\frac{a}{b} f(\zeta)\right] d \zeta .
$$

Taking the real part from both sides of the latter formula, one can express a solution of the Robin problem (17) in terms of the corresponding solutions to the Dirichlet and Neumann problems (18) and (19). Alternatively, one can divide formula (20) by $\mathrm{z}$ and then integrate, which results

$$
f(z)=f\left(z_{0}\right)+b\left(h(z)-h\left(z_{0}\right)\right)-a \int_{z}^{z_{0}} \frac{h(\zeta)}{\zeta} d \zeta-\int_{z}^{z_{0}} \frac{g(\zeta)}{\zeta} d \zeta .
$$

Assuming $\varphi_{u}=\varphi_{v}$ and taking into account formula (10), we obtain a Robin to Neumann operator

$$
v(z, \zeta)=v\left(z_{0}, \zeta_{0}\right)+\frac{b}{2}\left[w(z, \zeta)-w\left(z_{0}, \zeta_{0}\right)\right]-\frac{a}{2} \int_{z}^{z_{0}} \frac{w_{1}(\tau)}{\tau} d \tau-\frac{a}{2} \int_{\zeta}^{\zeta_{0}} \frac{w_{2}(\xi)}{\xi} d \xi,
$$

where $w(z, \zeta)=w_{1}(z)+w_{2}(\zeta)$.

Theorem 3. Let $\partial \mathbb{D}$ be the unit circle in the plane centered at the origin. If $w(z, \zeta)$ is a solution to (17), then a Robin to Neumann operator has the form

$$
v(z, \zeta)=v\left(z_{0}, \zeta_{0}\right)+\frac{b}{2}\left[w(z, \zeta)-w\left(z_{0}, \zeta_{0}\right)\right]-\frac{a}{2} \int_{z}^{z_{0}} \frac{w_{1}(\tau)}{\tau} d \tau-\frac{a}{2} \int_{\zeta}^{\zeta_{0}} \frac{w_{2}(\xi)}{\xi} d \xi .
$$

Here $v(z, \zeta)$ is a harmonic function such that $\frac{\partial v}{\partial n}=\varphi_{v}=\varphi_{w} / 2$ on $\partial \mathbb{D}$.

Proof. To prove formula (22) one needs to check the conditions in (19). Obviously, function $v(z, \zeta)$ is harmonic, $\frac{\partial^{2} v}{\partial z \partial \zeta}=0$ if $w(z, \zeta)$ is harmonic.

To check the second condition we differentiate (22) with respect to $r$,

$$
\frac{\partial}{\partial r} v(z, \zeta)=\frac{b}{2} \frac{\partial}{\partial r} w(z, \zeta)+\frac{a}{2} \frac{w_{1}(z)}{z} e^{i \theta}+\frac{a}{2} \frac{w_{2}(\zeta)}{z} e^{-i \theta} .
$$


Then on $\partial \mathbb{D}$, the latter formula reduces to

$$
\frac{\partial}{\partial r} v\left(z_{0}, \zeta_{0}\right)=\frac{b}{2} \frac{\partial}{\partial r} w\left(z_{0}, \zeta_{0}\right)+\frac{a}{2} \frac{w_{1}\left(z_{0}\right)}{e^{i \theta}} e^{i \theta}+\frac{a}{2} \frac{w_{2}\left(\zeta_{0}\right)}{e^{-i \theta}} e^{-i \theta}
$$

resulting in

$$
\frac{\partial}{\partial r} v\left(z_{0}, \zeta_{0}\right)=\varphi_{v}=\frac{b}{2} \frac{\partial}{\partial r} w\left(z_{0}, \zeta_{0}\right)+\frac{a}{2} w\left(z_{0}, \zeta_{0}\right)=\frac{1}{2} \varphi_{w}
$$

This finishes the proof.

Example 5.1. Consider a solution to Robin problem $w=\ln r$ satisfying the Robin condition with $\varphi_{w}=b$ on $\partial \mathbb{D}$. Function $w$ has the representation $w(z, \zeta)=w_{1}(z)+$ $w_{2}(\zeta)=\frac{1}{2} \ln z+\frac{1}{2} \ln \zeta$. Then formula (22) reads as

$$
v(z, \zeta)=v\left(z_{0}, \zeta_{0}\right)+\frac{b}{2} \ln r-\frac{a}{4} \int_{z}^{z_{0}} \frac{\ln (\tau)}{\tau} d \tau-\frac{a}{4} \int_{\zeta}^{\zeta_{0}} \frac{\ln (\xi)}{\xi} d \xi
$$

therefore,

$$
v(z, \zeta)=\frac{b}{2} \ln r+\frac{a}{4}\left(\ln ^{2} r-\theta^{2}\right)+\text { const },
$$

which is a harmonic function satisfying the Neumann condition on $\partial \mathbb{D}$ with $\varphi_{v}=\frac{b}{2}$.

Corollary 3.1. Solutions to the Dirichlet problem (18) are related to the solutions to the Robin problem (17),

$$
u=\frac{a}{2} w+\frac{b r}{2} \frac{\partial w}{\partial r}
$$

whenever $\varphi_{u}=\frac{1}{2} \varphi_{w}$ on $\partial \mathbb{D}$.

Indeed, formulae (10) and (22) imply

$$
\frac{b}{2}\left[w(z, \zeta)-w\left(z_{0}, \zeta_{0}\right)\right]-\frac{a}{2} \int_{z}^{z_{0}} \frac{w_{1}(\tau)}{\tau} d \tau-\frac{a}{2} \int_{\zeta}^{\zeta_{0}} \frac{w_{2}(\xi)}{\xi} d \xi=-\int_{z}^{z_{0}} \frac{u_{1}(\tau)}{\tau} d \tau-\int_{\zeta}^{\zeta_{0}} \frac{u_{2}(\xi)}{\xi} d \xi
$$

Assuming that $(x, y) \in \mathbb{R}^{2}$ and setting $z=r e^{i \theta}, \zeta=r e^{-i \theta}$, we have

$$
\frac{b}{2}\left[w\left(r e^{i \theta}, r e^{-i \theta}\right)-w\left(z_{0}, \zeta_{0}\right)\right]-\frac{a}{2} \int_{r}^{1} \frac{w\left(\rho e^{i \theta}, \rho e^{-i \theta}\right)}{\rho} d \rho=-\int_{r}^{1} \frac{u\left(\rho e^{i \theta}, \rho e^{-i \theta}\right)}{\rho} d \rho .
$$

Differentiation of the latter formula with respect to $r$ results in (23). 


\section{Reflection about a circular arc with nonhomogeneous Robin data}

To derive a reflection formula for solutions to (17) we use formula (14),

$$
v\left(\frac{e^{i \theta}}{r}, \frac{1}{r e^{i \theta}}\right)=v\left(r e^{i \theta}, r e^{-i \theta}\right)-\int_{\frac{1}{r}}^{r} \frac{\varphi_{v}\left(\rho e^{i \theta}, \frac{1}{\rho e^{i \theta}}\right)}{\rho} d \rho
$$

and formula (22),

$$
v(z, \zeta)=v\left(z_{0}, \zeta_{0}\right)+\frac{b}{2}\left[w(z, \zeta)-w\left(z_{0}, \zeta_{0}\right)\right]-\frac{a}{2} \int_{z}^{z_{0}} \frac{w_{1}(\tau)}{\tau} d \tau-\frac{a}{2} \int_{\zeta}^{\zeta_{0}} \frac{w_{2}(\xi)}{\xi} d \xi,
$$

which in the plane can be rewritten in variables $(r, \theta)$ as

$$
v\left(r e^{i \theta}, r e^{-i \theta}\right)=\frac{b}{2} w\left(r e^{i \theta}, r e^{-i \theta}\right)-\frac{a}{2} \int_{r}^{1} \frac{w\left(\rho e^{i \theta}, \rho e^{-i \theta}\right)}{\rho} d \rho+\widetilde{C},
$$

where $\widetilde{C}=v\left(z_{0}, \zeta_{0}\right)-\frac{b}{2} w\left(z_{0}, \zeta_{0}\right)$. This implies,

$$
v\left(\frac{e^{i \theta}}{r}, \frac{1}{r e^{i \theta}}\right)=\frac{b}{2} w\left(\frac{e^{i \theta}}{r}, \frac{1}{r e^{i \theta}}\right)-\frac{a}{2} \int_{1 / r}^{1} \frac{w\left(\rho e^{i \theta}, \rho e^{-i \theta}\right)}{\rho} d \rho+\widetilde{C},
$$

Making a substitution $\widetilde{\rho}=1 / \rho$ in the integral, we have

$$
v\left(\frac{e^{i \theta}}{r}, \frac{1}{r e^{i \theta}}\right)=\frac{b}{2} w\left(\frac{e^{i \theta}}{r}, \frac{1}{r e^{i \theta}}\right)+\frac{a}{2} \int_{r}^{1} \frac{w\left(\frac{1}{\tilde{\rho}} e^{i \theta}, \frac{1}{\tilde{\rho}} e^{-i \theta}\right)}{\widetilde{\rho}} d \widetilde{\rho}+\widetilde{C} .
$$

Plugging into formula (14), we obtain

$$
\begin{gathered}
\frac{b}{2} w\left(\frac{e^{i \theta}}{r}, \frac{1}{r e^{i \theta}}\right)+\frac{a}{2} \int_{r}^{1} \frac{w\left(\frac{1}{\rho} e^{i \theta}, \frac{1}{\rho} e^{-i \theta}\right)}{\widetilde{\rho}} d \widetilde{\rho}=\frac{b}{2} w\left(r e^{i \theta}, r e^{-i \theta}\right) \\
-\frac{a}{2} \int_{r}^{1} \frac{w\left(\rho e^{i \theta}, \rho e^{-i \theta}\right)}{\rho} d \rho-\int_{\frac{1}{r}}^{r} \frac{\varphi_{v}\left(\rho e^{i \theta}, \frac{1}{\rho e^{i \theta}}\right)}{\rho} d \rho,
\end{gathered}
$$

which leads to the following theorem.

Theorem 4. Let $w$ be a solution to the problem (17), then for a pair of points in the plain symmetric with respect to the unit circle centered at the origin, the following reflection formula holds

$$
w\left(\frac{e^{i \theta}}{r}\right)=w\left(r e^{i \theta}\right)-\frac{a}{b} \int_{r}^{1} \frac{w\left(\rho e^{i \theta}\right)+w\left(\frac{1}{\rho} e^{i \theta}\right)}{\rho} d \rho-\frac{1}{b} \int_{\frac{1}{r}}^{r} \frac{\varphi_{w}\left(\rho e^{i \theta}, \frac{1}{\rho e^{i \theta}}\right)}{\rho} d \rho .
$$


Example 6.1. Let $w$ be a solution to the problem (17) with $\varphi_{w}=b$. Then formula (24) reads as

$$
w\left(\frac{e^{i \theta}}{r}\right)=w\left(r e^{i \theta}\right)-\frac{a}{b} \int_{r}^{1} \frac{w\left(\rho e^{i \theta}\right)+w\left(\frac{1}{\rho} e^{i \theta}\right)}{\rho} d \rho-2 \ln r .
$$

Example 6.2. Let $w$ be a solution to the problem (17) with $\varphi_{w}=(a+2 b)\left(2 x^{2}-1\right)$. Taking into account that $\varphi_{w}=\frac{a+2 b}{2}\left(z^{2}+\zeta^{2}\right)$ on $\Gamma_{\mathbb{C}}$, formula (24) reduces to

$$
w\left(\frac{e^{i \theta}}{r}\right)=w\left(r e^{i \theta}\right)-\frac{a}{b} \int_{r}^{1} \frac{w\left(\rho e^{i \theta}\right)+w\left(\frac{1}{\rho} e^{i \theta}\right)}{\rho} d \rho-\frac{a+2 b}{2 b} \int_{\frac{1}{r}}^{r} \frac{\left.\rho^{2} e^{2 i \theta}+\frac{1}{\rho^{2} e^{2 i \theta}}\right)}{\rho} d \rho,
$$

which results in

$$
w\left(\frac{e^{i \theta}}{r}\right)=w\left(r e^{i \theta}\right)-\frac{a}{b} \int_{r}^{1} \frac{w\left(\rho e^{i \theta}\right)+w\left(\frac{1}{\rho} e^{i \theta}\right)}{\rho} d \rho-\frac{a+2 b}{2 b}\left(r^{2}-\frac{1}{r^{2}}\right) \cos (2 \theta) .
$$

Example 6.3. Let $w$ be a solution to the problem (17) with $\varphi_{w}=(a+b) \cos \theta$. Then the corresponding reflection formula is

$$
w\left(\frac{e^{i \theta}}{r}\right)=w\left(r e^{i \theta}\right)-\frac{a}{b} \int_{r}^{1} \frac{w\left(\rho e^{i \theta}\right)+w\left(\frac{1}{\rho} e^{i \theta}\right)}{\rho} d \rho-\frac{a+b}{2 b}\left(r-\frac{1}{r}\right) \cos \theta .
$$

\section{Dirichlet to Neumann operator and reflection about an arc of an algebraic curve}

In this section we generalize the results obtained for a circular arc in sections 3 and 4 for the case of an algebraic curve $\Gamma$. First, we discuss a Dirichlet to Neumann mapping. Then we apply this mapping for derivation of a reflection formula. Remark that this formula was obtained at [4], [5] using different methods.

Consider the following two problems for functions $u(x, y)$ and $v(x, y)$ defined near an arc of an algebraic curve $\Gamma$ in the plane:

$$
\begin{aligned}
& \left\{\begin{array}{l}
\Delta u=0 \\
u=\varphi
\end{array} \quad \text { on } \Gamma,\right. \\
& \left\{\begin{array}{l}
\Delta v=0 \\
\frac{\partial v}{\partial n}=\varphi
\end{array} \quad \text { near } \Gamma\right.
\end{aligned}
$$

where $\varphi$ is holomorphically continuable into $\mathbb{C}^{2}$ near $\Gamma$.

Theorem 5. Let $\Gamma$ be an arc of an algebraic curve in the plane. If $u(z, \zeta)$ is a solution to (25), then

$$
v(z, \zeta)=v\left(z_{0}, \zeta_{0}\right)+i \int_{z}^{z_{0}} u_{1}(\tau) \sqrt{S^{\prime}(\tau)} d \tau-i \int_{\zeta}^{\zeta_{0}} u_{2}(\xi) \sqrt{\widetilde{S}^{\prime}(\xi)} d \xi
$$


is a solution to (26). Here $u(z, \zeta)=u_{1}(z)+u_{2}(\zeta)$ is a harmonic function such that $u(z, \bar{z})=\varphi$ on $\Gamma$.

Proof. According to formula (27), function $v(z, \zeta)$ has a representation as a sum of a function $v_{1}(z)$ and a function $v_{2}(\zeta)$, and therefore, is harmonic, $\frac{\partial^{2} v}{\partial z \partial \zeta}=0$.

To check the second condition in (26), note that the normal derivative could be computed using the formula [2],

$$
\frac{\partial v}{\partial n}(z, \zeta)=\frac{i}{\sqrt{S^{\prime}(z)}}\left(\frac{\partial v_{1}}{\partial z}-\frac{\partial v_{2}}{\partial \zeta} S^{\prime}(z)\right)=\varphi .
$$

Differentiating formula (27), we have

$$
\frac{\partial v}{\partial n}(z, \zeta)=\frac{i}{\sqrt{S^{\prime}(z)}}\left(-i u_{1}(z) \sqrt{S^{\prime}(z)}-i u_{2}(\zeta) \sqrt{\widetilde{S}^{\prime}(\zeta)} S^{\prime}(z)\right),
$$

therefore, on the curve $\Gamma$ we obtain

$$
\frac{\partial v}{\partial n}(z, \zeta)_{\left.\right|_{\Gamma}}=\left(u_{1}(z)+u_{2}(\zeta)\right)_{\left.\right|_{\Gamma}}=u(z, \zeta)_{\left.\right|_{\Gamma}}=\varphi
$$

This finishes the proof.

Remark. For the special case when $\Gamma$ is the unit circle centered at the origin, $S(z)=$ $1 / z$, formula (27) reduces to formula (10).

To derive the reflection formula generalizing formula (14), let us rewrite formula 27) at the reflected point,

$$
v(\widetilde{S}(\zeta), S(z))=v\left(z_{0}, \zeta_{0}\right)+i \int_{\widetilde{S}(\zeta)}^{\widetilde{S}\left(\zeta_{0}\right)} u_{1}(\tau) \sqrt{S^{\prime}(\tau)} d \tau-i \int_{S(z)}^{S\left(z_{0}\right)} u_{2}(\xi) \sqrt{\widetilde{S}^{\prime}(\xi)} d \xi,
$$

and subtract (28) from (27),

$$
\begin{gathered}
v(z, \zeta)-v(\widetilde{S}(\zeta), S(z))=i \int_{z}^{z_{0}} u_{1}(\tau) \sqrt{S^{\prime}(\tau)} d \tau+i \int_{S(z)}^{S\left(z_{0}\right)} u_{2}(\xi) \sqrt{\widetilde{S}^{\prime}(\xi)} d \xi \\
-i \int_{\zeta}^{\zeta_{0}} u_{2}(\xi) \sqrt{\widetilde{S}^{\prime}(\xi)} d \xi-i \int_{\widetilde{S}(\zeta)}^{\tilde{S}\left(\zeta_{0}\right)} u_{1}(\tau) \sqrt{S^{\prime}(\tau)} d \tau .
\end{gathered}
$$

Making the substitution $\xi=S(\tau)$ in the second and third integrals

$$
i \int_{S(z)}^{S\left(z_{0}\right)} u_{2}(\xi) \sqrt{\widetilde{S^{\prime}}(\xi)} d \xi=i \int_{z}^{z_{0}} u_{2}(S(\tau)) \sqrt{S^{\prime}(\tau)} d \tau
$$




$$
-i \int_{\zeta}^{\zeta_{0}} u_{2}(\xi) \sqrt{\widetilde{S^{\prime}}(\xi)} d \xi=-i \int_{\widetilde{S}(\zeta)}^{\widetilde{S}\left(\zeta_{0}\right)} u_{2}(S(\tau)) \sqrt{S^{\prime}(\tau)} d \tau
$$

we have

$$
\begin{gathered}
v(z, \zeta)-v(\widetilde{S}(\zeta), S(z))=i \int_{z}^{z_{0}}\left[u_{1}(\tau)+u_{2}((S(\tau))] \sqrt{S^{\prime}(\tau)} d \tau\right. \\
-i \int_{\widetilde{S}(\zeta)}^{\widetilde{S}\left(\zeta_{0}\right)}\left[u_{1}(\tau)+u_{2}(S(\tau))\right] \sqrt{S^{\prime}(\tau)} d \tau .
\end{gathered}
$$

Taking into account that $u_{1}(z)+u_{2}(S(z))=u(z, S(z))=\varphi(z, S(z))$ and $z_{0}=\widetilde{S}\left(\zeta_{0}\right)$, we arrive at the formula generalizing (14),

$$
v(\widetilde{S}(\zeta), S(z))=v(z, \zeta)+i \int_{\widetilde{S}(\zeta)}^{z} \varphi(\tau, S(\tau)) \sqrt{S^{\prime}(\tau)} d \tau
$$

\section{References}

[1] L. Beznea, M.N. Pascu, N.R. Pascu, An equivalence between the Dirichlet and the Neumann problem for the Laplace operator, Potential Anal., 44, 655-672 (2016).

[2] Ph. Davis, The Schwarz function and its applications, Carus Mathematical Monographs, MAA (1979).

[3] T. Savina, "On non-local reflection for elliptic equation of the second order in $\mathbb{R}^{2}$ (the Dirichlet condition)", Transactions of the American Mathematical Society, 364 (2012), no. 5, 2443-2460.

[4] T.V. Savina, "A reflection formula for the Helmholtz equation with the Neumann condition", Comput. Math. Math. Phys. 39 (1999), no. 4, 652-660.

[5] T. Savina, "From reflections to elliptic growth", http://arxiv.org/abs/1807.09903

[6] E. Study, Einige elementare Bemerkungen uber den Prozess der analytischen Fortsetzung, Math. Ann., 63, 239-245 (1907). 